\title{
A Model of Wavefunction Collapse in Discrete Space-Time
}

\author{
Gao Shan ${ }^{1}$ \\ Published Online: May 26, 2006
}

\begin{abstract}
We give a new argument supporting a gravitational role in quantum collapse. It is demonstrated that the discreteness of space-time, which results from the proper combination of quantum theory and general relativity, may inevitably result in the dynamical collapse of the wave function. Moreover, the minimum size of discrete space-time yields a plausible collapse criterion consistent with experiments. By assuming that the source to collapse the wave function is the inherent random motion of particles described by the wave function, we further propose a concrete model of wavefunction collapse in the discrete space-time. It is shown that the model is consistent with the existing experiments and macroscopic experiences.
\end{abstract}

KEY WORDS: discrete space-time; gravity; wavefunction collapse; random motion of particles.

PACS numbers: 0365B; 0460.

\section{INTRODUCTION}

Quantum measurement problem is the fundamental problem of quantum theory. The theory does not tell us how and when the measurement result appears. The projection postulate is just a makeshift (Bell, 1993). In this sense, the existing quantum theory is an incomplete description of the realistic process. Therefore it is natural to consider the continuous Schrödinger evolution and the discontinuous quantum collapse as two ideal approximations of a unified evolution process. The new theory describing such unified evolution is generally called revised quantum dynamics or dynamical collapse theory. It has been widely studied in recent years (Ghirardi et al., 1986; Diosi et al., 1989; Pearle, 1989; Ghirardi et al., 1990; Percival, 1994; Hughston, 1996; Penrose, 1996; Adler et al., 2001; Shan, 2000, 2003).

An important problem of revised quantum dynamics is the origin of quantum collapse. It may be very natural to guess that the collapse of wave function is

\footnotetext{
${ }^{1}$ Institute of Electronics, Chinese Academy of Sciences, LongZeYuan 24-3-501, ChangPing District,
} Beijing 102208, P.R. China; e-mail: rg@mail.ie.ac.cn. 
induced by gravity. The reasons include: (1) gravity is the only universal force being present in all physical interactions; (2) gravitational effects grow with the size of the objects concerned, and it is in the context of macroscopic objects that linear superpositions may be violated. The gravity-induced collapse conjecture can be traced to Feynman (1995). In his Lectures on Gravitation, he considers the philosophical problems in quantizing macroscopic objects and contemplates on a possible breakdown of quantum theory. He said, "I would like to suggest that it is possible that quantum mechanics fails at large distances and for large objects, ... it is not inconsistent with what we do know. If this failure of quantum mechanics is connected with gravity, we might speculatively expect this to happen for masses such that $G M^{2} / \hbar c=1$, of $M$ near $10^{-5}$ g."

Penrose further strengthened the gravity-induced collapse argument (Penrose, 1996). He argued that the superposition of different space-times is physically improper, and the evolution of such superposition can not be defined in a consistent way. This requires that a quantum superposition of two space-time geometries, which corresponds to two macroscopically different energy distributions, should collapse after a very short time. Penrose's argument reveals a profound and fundamental conflict between the general covariance principle of general relativity and the superposition principle of quantum mechanics. According to general relativity, there exists one kind of dynamical connection between motion and space-time, i.e., the motion of particles is defined in space-time, at the same time, space-time is determined by the motion of particles. Then when we consider the superposition state of different positions of a particle, say position $A$ and position $B$, one kind of logical inconsistency appears. On the one hand, according to quantum theory, the valid definition of such superposition requires the existence of a definite spacetime background, in which the position $A$ and position $B$ can be distinguished. On the other hand, according to general relativity, the space-time, including the distinguishability of the position $A$ and position $B$, can not be predetermined, and must be dynamically determined by the position superposition state. Since the different position states in the superposition determine different space-times, the space-time determined by the whole superposition state is indefinite. Then an essential conflict between quantum theory and general relativity does appear. Penrose believed that the conflict requires that the quantum superposition of different space-times can not exist in a precise way, and should collapse after a very short time. Thus gravity may indeed be the physical origin of wavefunction collapse.

In this paper, we will give a new argument supporting a gravitational role in quantum collapse. It is demonstrated that the discreteness of space-time, which results from the proper combination of quantum theory and general relativity, may inevitably result in the dynamical collapse of wave function. Moreover, the minimum size of discrete space-time indeed yields a plausible collapse criterion consistent with experiments. This analysis reinforces and completes Penrose's argument. By assuming that the source to collapse the wave function is the inherent 
random motion of particles described by the wave function, we further propose a concrete model of quantum collapse in the discrete space-time. It is shown that the model is consistent with the existing experiments and macroscopic experiences.

\section{DISCRETE SPACE-TIME AND ITS IMPLICATIONS FOR QUANTUM COLLAPSE}

Quantum theory and general relativity are both based on the continuous spacetime assumption. However, the appearance of infinity in quantum field theory and singularity in general relativity has implied that space-time may be not continuous but discrete. In fact, it has been widely argued that the proper combination of quantum theory and general relativity may inevitably result in the discreteness of space-time (Einstein, 1936; Snyder, 1947; Heisenberg, 1957; Salecker and Wigner, 1958; Garay, 1995; Rovelli and Smolin, 1995; Polchinski, 1998; Adler and Santiago, 1999; Amelino-Camelia, 2000; Smolin, 2001). For example, the discreteness of space-time can be deduced from the following generalized uncertainty principle (GUP) (Garay, 1995; Adler and Santiago, 1999):

$$
\Delta x=\Delta x_{Q M}+\Delta x_{G R} \geq \frac{\hbar}{2 \Delta p}+\frac{2 L_{p}^{2} \Delta p}{\hbar}
$$

In the discrete space-time, there exist a minimum time interval $T_{U} \equiv 2 T_{P}$ and a minimum length $L_{U} \equiv 2 L_{P}$, where $T_{P}=\left(\frac{G \hbar}{c^{5}}\right)^{1 / 2}, L_{P}=\left(\frac{G \hbar}{c^{3}}\right)^{1 / 2}$ are the Planck time and Planck length. The physical meaning of such discrete space-time is that any space-time difference smaller than the minimum time interval and minimum length is in principle undetectable, i.e., the space-times with a difference smaller than the minimum sizes are physically identical. It should be noted that the discreteness of space-time is essentially one kind of quantum properly due to the universal quantum fluctuations of space-time, and thus the space-times with a difference smaller than the minimum sizes are not absolutely identical, but nearly identical.

In the following, we will argue that the discreteness of space-time may inevitably result in the dynamical collapse of wave function. The argument also gives a plausible criterion of quantum collapse in the discrete space-time. We will first point out one deficiency in Penrose's argument. As we think, His argument may inevitably fail in the continuous space-time. If space-time is continuous, then the space-times with any small difference are physically different. Since the quantum superposition of different space-times is physically ill defined due to the conflict between general relativity and quantum mechanics, such state can not exist in reality. Thus the quantum superposition of two space-times with a very small difference such as the superposition states of microscopic particles can not exist either. This does not accord with experiment. However, if space-time is discrete, and there exist a minimum time interval and a minimum length, then 
Penrose's argument can be reinforced and thus succeed. In short, the discreteness of space-time may cure the above deficiency in Penrose's argument. The key point is that two space-times with a difference smaller than the minimum sizes are the physically same space-times in the discrete space-time. Thus their quantum superposition can exist and collapse after a finite time interval. Only the quantum superposition of two space-times with a difference larger than the minimum sizes can not exist, and should collapse instantaneously. Such dynamical collapse of wave function can accord with experiment.

In order to make our prescription be precise, we need to define the difference between two space-times. As indicated by the generalized uncertainty principle, namely the Equation (1), the difference of energy $\Delta E$ corresponds to the difference of space-time $\frac{2 L_{p}^{2} \Delta E}{\hbar c}$. Then as to the two states in the quantum superposition with energy difference $\Delta E$, the difference between the space-times determined by the states may be characterized by the quantity $\frac{2 L_{p}^{2} \Delta E}{\hbar c}$. The physical meaning of such space-time difference can be clarified as follows. Let the two energy eigenstates in the superposition be limited in the regions with the same radius $R$ (they may locate in different positions). Then the space-time outside the region can be described by the Schwarzschild metric:

$$
d s^{2}=\left(1-\frac{r_{S}}{r}\right)^{-1} d r^{2}+r^{2} d \theta^{2}+r^{2} \sin \theta^{2} d \phi^{2}-\left(1-\frac{r_{S}}{r}\right) c^{2} d t^{2}
$$

where $r_{S}=\frac{2 G E}{c^{4}}$ is the Schwarzschild radius. By assuming that the metric tensor inside the region $R$ is the same as that in the boundary, the proper size of the region is

$$
L=2 \int_{0}^{R}\left(1-\frac{r_{S}}{R}\right)^{-1 / 2} d r
$$

Then the space difference of the two space-times in the superposition inside the region $R$ can be characterized by

$$
\Delta L \approx \int_{0}^{R} \frac{\Delta r_{S}}{R} d r=\Delta r_{S}=\frac{2 L_{p}^{2} \Delta E}{\hbar c}
$$

This result is consistent with the generalized uncertainty principle. Thus as to the two states in the quantum superposition, we can define the difference of their corresponding space-times as the difference of the proper spatial sizes of the regions occupied by the states. Such difference represents the fuzziness of the point-by-point identification of the spatial section of the two space-times. As a result, the space-translation operators are not the same for the two space-times. In comparison with Penrose's definition of acceleration uncertainty, our definition 
may be taken as some kind of position uncertainty in the superposition of spacetimes. Such uncertainty does not depend on the spatial distance between the states in the superposition. A detailed comparison of them will be given in the next section.

The space-time difference defined above can be re-written as the following form:

$$
\frac{\Delta L}{L_{U}} \approx \frac{\Delta E}{E_{P}}
$$

where $E_{p}=h / T_{p}$ is the Planck energy. This relation seems to indicate some kind of equivalence between the difference of energy and the difference of spacetimes defined above. However, it should be stressed that they are not equivalent for the general situations. In physics, it is the difference of space-times, not the energy difference in the superposition that results in the dynamical collapse of wave function. In addition, the proper size of the region occupied by the state is not solely determined by the energy of the state, but determined by the energy distribution of all entangled states. The latter determines the metric tensor inside the region. For example, as to the entanglement state such as $\psi_{1} \varphi_{1}+\psi_{2} \varphi_{2}$, the difference of the proper sizes of the regions occupied by the states $\psi_{1}$ and $\psi_{2}$ is also influenced by the energy distribution of the entangled states $\varphi_{1}$ and $\varphi_{2}$. Some concrete examples will be given in the Section 4.

Now we can give a collapse criterion in terms of the above analysis. If the difference $\Delta L$ of the space-times in the superposition equals to or is larger than the minimum size $L_{U}$, the superposition state will collapse to one of the definite spacetimes instantaneously. If the difference $\Delta L$ of the space-times in the superposition is smaller than $L_{U}$, the superposition state will collapse after a finite time interval. Thus the superposition of space-times can only possess a space-time uncertainty smaller than the minimum size in the discrete space-time. If such uncertainty limit is exceeded, the superposition will collapse to one of the definite space-times instantaneously.

Lastly, we note that the above collapse criterion is also consistent with the requirement of discrete space-time. This can be seen from the analysis of a typical example. Consider a quantum superposition of two energy eigenstates. The initial state is

$$
\psi(x, 0)=\frac{1}{\sqrt{2}}\left[\varphi_{1}(x)+\varphi_{2}(x)\right]
$$

where $\varphi_{1}(x)$ and $\varphi_{2}(x)$ are two energy eigenstates with the energy eigenvalues $E_{1}$ and $E_{2}$. According to the linear Schrödinger evolution, we have:

$$
\psi(x, t)=\frac{1}{\sqrt{2}}\left[e^{-i E_{1} t / \hbar} \varphi_{1}(x)+e^{-i E_{2} t / \hbar} \varphi_{2}(x)\right]
$$


and

$$
\rho(x, t)=|\psi(x, t)|^{2}=\frac{1}{2}\left[\varphi_{1}^{2}(x)+\varphi_{2}^{2}(x)+2 \varphi_{1}(x) \varphi_{2}(x) \cos (\Delta E t / \hbar)\right]
$$

This result indicates that the probability density $\rho(x, t)$ will oscillate with a pe$\operatorname{riod} T=h / \Delta E$ in each position of space, where $\Delta E=E_{2}-E_{1}$ is the energy difference. When the energy difference $\Delta E$ exceeds the Planck energy $E_{P}$, the difference of the space-times determined by the energy eigenstates will be larger than the minimum size $L_{U}$, and the superposition state can not exist according to the above collapse criterion. This means that the probability density $\rho(x, t)$ can not oscillate with a period shorter than the minimum time interval $T_{U}$. This result is consistent with the requirement of discrete space-time. In the discrete space-time, the minimum time interval $T_{U}$ is the minimum distinguishable size of time, and no change can happen during a time interval shorter than $T_{U}$.

\section{A MODEL OF QUANTUM COLLAPSE IN DISCRETE SPACE-TIME}

It is well known that a chooser and a choice are needed to bring the required dynamical collapse of wave function (Pearle, 1999). According to the above analysis, the choice should be the energy distribution that determines the spacetime geometry. Then who is the chooser? In this section, we will try to solve the chooser problem. A concrete model of quantum collapse in discrete space-time will also be proposed in terms of the new chooser.

In the usual wavefunction collapse models, the chooser is generally an unknown random classical field. However, such models may have some inherent problems concerning the chooser. For example, when the classical field is quantized, its collapse will need another random classical field. The process is an infinite chain, which is very similar to the von Neumann's infinite chains of measurement (von Neumann, 1955). As a result, the models can not explain where the intrinsic randomness originates. In order to cut the infinite chain, it seems more reasonable that the randomness of the collapse process originates from the wave function itself. It has been argued that the probability relating to the wave function is not only the display of the measurement results, but also the objective character of the motion of particles (Bunge, 1973; Shimony, 1993; Shan, 2000, 2003). Thus it is not unreasonable to assume that the motion of particles described by the wave function is an intrinsic random process. Then the collapse of wave function may result from such random motion of particles in space-time, i.e., the chooser is the random motion of particles described by the wave function. In such a model, the motion of particles naturally provides a random source to collapse the wave function describing the motion, and the dynamical collapse of wave function may be the inherent display of such random motion of particles. This point of view is natural and simple. It may not only help to explain the collapse of 
wave function, but also physically explain the wave function, which is taken as the mathematical description of the random motion of particles (Shan, 2000, 2003).

In accordance with the assumption that the measurement results should reflect the measured realistic process, the stochastic distribution of the random motion of particle will be the same as the probability distribution of measurement results, which satisfies the Born's rule in quantum mechanics. Thus we have:

$$
p(A, t)=P(A, t)
$$

where $A$ is a property of the random motion of particle such as position, momentum and energy etc, $P(A, t)$ is the probability distribution of the measurement results of $A$ in quantum mechanics, and $p(A, t)$ is the probability distribution of the property $A$ of the particle in random motion. This is the basic character of the random motion of particles described by the wave function.

In the following, we will analyze the influence of the random motion to the wave function, and try to work out the collapse law of wave function. As a typical example, we analyze a simple two-level system which initial state is

$$
|\psi, 0\rangle=\sqrt{P_{1}(0)}\left|E_{1}\right\rangle+\sqrt{P_{2}(0)}\left|E_{2}\right\rangle
$$

where $\left|E_{1}\right\rangle$ and $\left|E_{2}\right\rangle$ are two energy eigenstates with eigenvalues $E_{1}$ and $E_{2}, P_{1}(0)$ and $P_{2}(0)$ are the corresponding probability which satisfy the conservation relation $P_{1}(0)+P_{2}(0)=1$. Since the linear Schrödinger evolution does not change the probability distribution, we can only consider the influence of dynamical collapse on the probability distribution. As to the random motion of particle described by the above state, the energy of particle assumes $E_{1}$ or $E_{2}$ in a random way, and the corresponding probability is respectively $P_{1}(0)$ and $P_{2}(0)$ at the initial instant. In other words, the particle is in the state $\left|E_{1}\right\rangle$ with the probability $P_{1}(0)$, and is in the state $\left|E_{2}\right\rangle$ with the probability $P_{2}(0)$ at the initial instant. In the discrete space-time, this means that at the initial instant the particle stays in the state $\left|E_{1}\right\rangle$ for a time unit $T_{U} \equiv 2 T_{P}$ with the probability $P_{1}(0)$, and stays in the state $\left|E_{2}\right\rangle$ for a time unit $T_{U} \equiv 2 T_{P}$ with the probability $P_{2}(0)$.

Assume after the particle stays in the state $\left|E_{1}\right\rangle$ for a time unit $T_{U}, P_{1}(t)$ turns to be

$$
P_{11}\left(t+T_{U}\right)=P_{1}(t)+\Delta P_{1}
$$

where $\Delta P_{1}$ is a functional of $P_{1}(t)$. Then considering the conservation of probability, $P_{2}(t)$ turns to be

$$
P_{21}\left(t+T_{U}\right)=P_{2}(t)-\Delta P_{1}
$$

The probability of such stay is $p\left(E_{1}, t\right)=P_{1}(t)$. Accordingly, we assume after the particle stays in the state $\left|E_{2}\right\rangle$ for a time unit $T_{U}, P_{2}(t)$ turns to be

$$
P_{22}\left(t+T_{U}\right)=P_{2}(t)+\Delta P_{2}
$$


then $P_{1}(t)$ turns to be

$$
P_{12}\left(t+T_{U}\right)=P_{1}(t)-\Delta P_{2}
$$

The probability of such stay is $p\left(E_{2}, t\right)=P_{2}(t)$.

Then we can work out the diagonal density matrix elements of the evolution:

$$
\begin{aligned}
\rho_{11}\left(t+T_{U}\right) & =\sum_{i=1}^{2} p\left(E_{i}, t\right) P_{1 i}\left(t+T_{U}\right) \\
& =P_{1}(t)\left[P_{1}(t)+\Delta P_{1}\right]+P_{2}(t)\left[P_{1}(t)-\Delta P_{2}\right] \\
& =P_{1}(t)+\left[P_{1}(t) \Delta P_{1}-P_{2}(t) \Delta P_{2}\right] \\
& =\rho_{11}(t)+\left[P_{1}(t) \Delta P_{1}-P_{2}(t) \Delta P_{2}\right] \\
\rho_{22}\left(t+T_{U}\right) & =\rho_{22}(t)+\left[P_{2}(t) \Delta P_{2}-P_{1}(t) \Delta P_{1}\right]
\end{aligned}
$$

Since the probability distribution of the collapse results should satisfy the Born's rule in quantum mechanics, we require $\rho_{11}\left(t+T_{U}\right)=\rho_{11}(t)$ and $\rho_{22}\left(t+T_{U}\right)=$ $\rho_{22}(t)$. Then we can obtain the relation:

$$
P_{1} \Delta P_{1}-P_{2} \Delta P_{2}=0
$$

When the superposition state contains more than two branches, the above requirement will lead to the following equations set:

$$
\Delta P_{i}-\sum_{j \neq i} \frac{P_{j} \Delta P_{j}}{1-P_{j}}=0
$$

where $\sum_{i} P_{i}=1$, and $i, j$ denotes the branch states. Here we assume that the increase $\Delta P_{i}$ of one branch comes from the scale-down of the other branches, where the scale is the probability $P_{j}$ of each of these branches. By solving this equations set, we find the following solution:

$$
\Delta P_{i}=k\left(1-P_{i}\right)
$$

where $k$ is an undetermined dimensionless quantity. This is an important relation describing the dynamical collapse of wave function in the discrete space-time.

By using the above relation, we can further work out the non-diagonal density matrix elements of the evolution:

$$
\begin{aligned}
\rho_{12}\left(t+T_{U}\right)= & \sum_{i=1}^{2} p\left(E_{i}, t\right) \sqrt{P_{1 i}\left(t+T_{U}\right)} \sqrt{P_{2 i}\left(t+T_{U}\right)} \\
= & P_{1}(t) \sqrt{P_{1}(t)+\Delta P_{1}} \sqrt{P_{2}(t)-\Delta P_{1}} \\
& +P_{2}(t) \sqrt{P_{1}(t)-\Delta P_{2}} \sqrt{P_{2}(t)+\Delta P_{2}}
\end{aligned}
$$




$$
\begin{aligned}
& \approx\left(1-\frac{1}{4} k^{2}\right) \rho_{12}(t) \\
\rho_{21}\left(t+T_{U}\right) & \approx\left(1-\frac{1}{4} k^{2}\right) \rho_{21}(t)
\end{aligned}
$$

Then we have:

$$
\rho_{12}(t) \approx\left[1-\frac{1}{4} k^{2}\right]^{t / T_{U}} \rho_{12}(0)
$$

Let $\rho_{12}(t)=\frac{1}{2} \rho_{12}(0)$, we can get the appropriate collapse time formula:

$$
\tau_{c} \approx 2 k^{-2} T_{U}
$$

According to the collapse criterion obtained in the last section, the factor $k$ is a functional of the space-time difference $\Delta L$. When $\Delta L=0$, collapse never happens, thus we have $\Delta P_{i}=0, k=0$; when $\Delta L=L_{U}$, collapse happens instantaneously, thus we have $\Delta P_{i}=1-P_{i}, k=1$. Then when assuming the general differentiability of the function $k(\Delta L)$ and considering the dimensional relation we can obtain:

$$
k(\Delta L)=\sum_{i=1}^{\infty} k^{(i)}(0)\left(\frac{\Delta L}{L_{U}}\right)^{i}
$$

When $k^{(1)}(0)=0$ and $k^{(2)}(0) \neq 0$, the collapse time $\tau_{c} \approx\left(\frac{L_{p}}{\Delta L}\right)^{4} T_{p}$ is too long for some situations and contradicts the experiments (Ghirardi et al., 1990; Pearle, 1999). Thus we can get the factor $k$ in the first rank:

$$
k=\frac{\Delta L}{L_{U}}
$$

Here we omit the dimensionless constant $k^{(1)}(0)$ which is generally in the level of one. Then the collapse time formula is:

$$
\tau_{c} \approx 2\left(\frac{L_{U}}{\Delta L}\right)^{2} T_{U}
$$

During the dynamical collapse process, when the particle stays in the state $\left|E_{i}\right\rangle$ for a time unit $T_{U}, P_{i}(t)$ turns to be

$$
\Delta P_{i}(t)=\frac{\Delta L}{L_{U}}\left[1-P_{i}(t)\right]
$$

By using the equivalent relation (5) for the superposition state of two energy eigenstates, we can re-write the above formulae as follows:

$$
\tau_{c} \approx \frac{2 \hbar E_{P}}{(\Delta E)^{2}}
$$




$$
\Delta P_{i}(t)=\frac{\Delta E}{E_{P}}\left[1-P_{i}(t)\right]
$$

For a general energy superposition state, $\Delta E$ may be defined as the squired energy uncertainty of the state, i.e.

$$
\Delta E=\left[\sum_{i} P_{i}\left(E_{i}-\bar{E}\right)^{2}\right]^{1 / 2}
$$

where $\bar{E}=\sum_{i} P_{i} E_{i}$ is the average energy of the state. As a result, the collapse time will generally relate to the initial energy probability distribution of the state.

We have two comments on the above collapse formula. First, even though the formula is the same as that in the energy-driven collapse model for some special situations such as the above energy superposition state (Hughston, 1996; Adler et al., 2001), our collapse model is essentially different from the energy-driven collapse model. In our model, the choice is the energy distribution, while the choice is the whole energy in the energy-driven collapse model. This has been stressed in the definition and the analysis of $\Delta L$ in the last section. Such difference can also be clearly seen in the common position measuring situation, which will be discussed in the next section. The energy-driven collapse model can not account for the appearance of definite macroscopic measurement results (Pearle, 2004), while our collapse model can do.

Next, we give an analysis of the relation between the above collapse formula and that proposed by Penrose. In Penrose's gravity-induced collapse model (Penrose, 1996), the collapse time formula is $\tau_{c} \approx \frac{\hbar}{\Delta E_{G}}$, where $\Delta E_{G}$ is the gravitational self-energy of the difference between the mass distributions belonging to the two states in the superposition

$$
\Delta E_{G}=\frac{1}{G} \int\left(\nabla \Phi_{2}-\nabla \Phi_{1}\right)^{2} d x^{3}
$$

where $\Phi_{1}$ and $\Phi_{2}$ are the Newtonian gravitational potentials of the two states, and $G$ is Newton's gravitational constant. When the two states in the superposition are in the same spatial region with radius $R$, we have

$$
\begin{aligned}
\Delta E_{G} & \approx \frac{G(\Delta E)^{2}}{c^{4} R} \\
\tau_{c} & \approx \frac{\hbar}{\Delta E_{G}} \approx \frac{c^{4} \hbar R}{G(\Delta E)^{2}}=\frac{1}{\frac{L_{P}}{R}\left(\frac{\Delta E}{E_{P}}\right)^{2}} T_{P}
\end{aligned}
$$

In our collapse model this requires that $k \approx \sqrt{\frac{L_{P}}{R}} \frac{\Delta E}{E_{P}}$. This term comes from some kind of acceleration uncertainty in the superposition of space-times according to 
Penrose's analysis. In comparison, our choice $k \approx \frac{\Delta E}{E_{p}}$ comes from the position uncertainty in the superposition of space-times. We may consider the former as a $1 / 2$ order $O\left(L_{P}^{1 / 2}\right)$ correction of the latter. Its existence also implies that a term of zero order $O(1) \approx \frac{\Delta E}{E_{P}}$ may exist. In fact, the sole existence of Penrose's term will contradict the discreteness of space-time as implied from the analysis in the last section. Since Penrose's term is extremely smaller than ours in most situations where $R \gg L_{P}$, it can be omitted in our collapse model. In addition, we note that Penrose's collapse time formula seems to be not right for the situations where the two energy eigenstates in the superposition are in the different spatial regions. His formula predicts that such superposition should collapse in the Newtonian limit, but it has been reasonably argued that the superposition does not collapse (Christian, 2001).

Lastly, we will stress some interesting characters of the above collapse model. First, the collapse dynamics in the model can be taken as a modified version of Pearle's (1999) gambler's ruin game dynamics. It has some new reasonable characters. For example, the collapse process proceeds gradually all the time in the model. Especially when the collapse process approaches completion, the random motion of particles still changes the whole probability distribution gradually. Whereas in the existing collapse models (Pearle, 1999), the assumed noise changes the whole probability distribution very largely when the collapse process approaches completion, even though such change happens in a very small probability. This way of change seems very unnatural. Secondly, the evolution law of wavefunction collapse can be uniquely determined by the assumed random motion of particles in our collapse model. The uniqueness of the collapse law may imply the validity of the collapse model. Lastly, our model is essentially discrete, and has no corresponding formulation in the continuous space-time. Its validity strongly relies on the discreteness of space-time, which is generally taken as an indispensable element in a complete theory of quantum gravity.

\section{SOME CONSIDERATIONS ON THE CONSISTENCY WITH EXPERIMENTS}

In our collapse model, the preferred bases are the energy eigenstates, namely the stationary solutions of Schrödinger equation. Such states correpond to the definite space-time geometries. This is not inconsistent with the microscopic experiments. Even though there is large spatial spreading for the energy eigenstates, their superposition may have very small spatial spreading. Since the energy uncertainty of such superposition can be very small, its collapse time will be very long. Thus the quantum state with small spatial spreading can still hold throughout the duration of usual experiments. For example, as to a quantum superposition state with spatial spreading $\Delta x \approx 0.1 \mu \mathrm{m}$, its energy uncertainty can be as small as 
$\Delta E \approx 1 \mathrm{eV}$ when satisfying the Heisenberg uncertainty relation, and the collapse time is $\tau_{c} \approx 10^{12} \mathrm{~s}$.

In addition, our collapse model does not contradict the macroscopic experiences either. The environmental influence will result in the large energy uncertainty in the quantum superposition of localized states, and thus collapse the superposition to the localized states very soon. As a result, the macroscopic objects can be always localized due to the environmental influence (for a detailed analysis see Adler, 2001). For example, for a common object of size $10^{-8} \mathrm{~cm}$ in the atmosphere at standard temperature and pressure, one nitrogen molecule accretes in the object during a time interval of $10^{-8} \mathrm{~s}$ in average (Redhead, 1996; Adler, 2001). Thus the energy uncertainty resulting from the accretion fluctuation is $\Delta E \approx 28 \mathrm{GeV}$ (corresponding to the mass of a nitrogen molecule) for a superposition of two localized states of object separated by the distance $10^{-8} \mathrm{~cm}$, and such superposition will collapse to one of the localized states after a time $\tau_{c} \approx 10^{-8} \mathrm{~s}$.

In the following, we give two typical examples concerning the application of our collapse model. In the first example, we consider an initial state describing a particle in a superposition of two locations (e.g. a superposition of two gaussian wavepacket separated by a certain distance). After the measurement interaction, the position measuring apparatus evolves to a superposition of macroscopically distinguishable states:

$$
\left(c_{1} \psi_{1}+c_{2} \psi_{2}\right) \varphi_{0} \rightarrow c_{1} \psi_{1} \varphi_{1}+c_{2} \psi_{2} \varphi_{2}
$$

where $\psi_{1}, \psi_{2}$ are the states of the particle in different locations, $\varphi_{0}$ is the initial state of the position measuring apparatus, and $\varphi_{1}, \varphi_{2}$ are the different outcome states of the apparatus. For an ideal measurement, the two particle/apparatus states $\psi_{1} \varphi_{1}$ and $\psi_{2} \varphi_{2}$ have precisely the same energy spectrum (Pearle, 2004). However, since the different measurement results appear in different positions of the apparatus, the two particle/apparatus states do possess different energy distribution. For example, the different position states of the photon in a superposition are detected in the different positions of the photographic plate, and they interact with the different $\mathrm{AgCl}$ molecules in these positions. Thus we should rewrite the apparatus states as $\varphi_{0}=$ $\chi_{A}(0) \chi_{B}(0), \varphi_{1}=\chi_{A}(1) \chi_{B}(0), \varphi_{2}=\chi_{A}(0) \chi_{B}(1)$, where $\chi_{A}(0), \chi_{B}(0)$ denote the initial states of the apparatus in the positions $A$ and $B, \chi_{A}(1), \chi_{B}(1)$ denote the outcome states of the apparatus in the positions $A$ and $B$. Such description clearly shows that different outcome states of the apparatus possess different energy distributions. Then we have

$$
\left(c_{1} \psi_{1}+c_{2} \psi_{2}\right) \chi_{A}(0) \chi_{B}(0) \rightarrow c_{1} \psi_{1} \chi_{A}(1) \chi_{B}(0)+c_{2} \psi_{2} \chi_{A}(0) \chi_{B}(1)
$$

Since there always exists some kind of measurement amplification from the microscopic state to the macroscopic outcome in the common measurement process, there is a big energy difference between the states $\chi_{A}(0), \chi_{B}(0)$ and $\chi_{A}(1), \chi_{B}(1)$. This means that the apparatus states in the superposition possess 
very different energy distribution in the positions $A$ and $B$, and the space-times in the superposition are also very different in these positions. Such difference will result in the proper quantum collapse in the measurement process according to our collapse model. As a typical example, as to the single photon detectoravalanche photodiodes, the energy consumption is sharply peaked in the very short measuring intervals (Berg, 1996). One type of avalanche photodiode operates at $10^{5} \mathrm{cps}$ and has a mean power dissipation of $4 \mathrm{~mW}$ (Berg, 1996; Cova et al., 1996). This corresponds to an energy consumption of about $2.5 \times 10^{11} \mathrm{eV}$ per measuring interval $10^{-5} \mathrm{~s}$. By using the collapse time formula $\tau_{c} \approx \frac{\hbar E_{P}}{(\Delta E)^{2}}$, where the energy difference $\Delta E$ between the states such as $\chi_{A}(0)$ and $\chi_{A}(1)$ is $\Delta E \approx$ $2.5 \times 10^{11} \mathrm{eV}$, we find that the collapse time is $\tau_{c} \approx 1.25 \times 10^{-10} \mathrm{~s}$. This time scale is smaller than and close to the measuring interval. Thus our collapse model is consistent with the experiments, and can account for the appearance of definite macroscopic measurement results. In addition, the measurement parameters of avalanche photodiodes may have provided an indirect confirmation of the model.

In the second example, we consider the $K_{L}^{0}$ meson decay process. The state of $K_{L}^{0}$ meson can be written as follows:

$$
\left|K_{L}^{0}\right\rangle=\frac{1}{\sqrt{2}}\left(\left|K^{0}\right\rangle-\left|\bar{K}^{0}\right\rangle\right)=\frac{1}{\sqrt{2}}(|s\rangle|\bar{d}\rangle-|d\rangle|\bar{s}\rangle)
$$

This is an entanglement state between the two level systems $|d\rangle,|s\rangle$ and $|\bar{d}\rangle,|\bar{s}\rangle$. Similarly, it seems that the energy difference between the two states $|s\rangle|\bar{d}\rangle$ and $|d\rangle|\bar{s}\rangle$ should be the effective mass difference of the $s$ and $d$ quarks, which is about $\Delta E \approx 100 \mathrm{MeV}$. Then the state $\left|K_{L}^{0}\right\rangle$ will collapse to the state $\left|K^{0}\right\rangle$ or $\left|\bar{K}^{0}\right\rangle$ during a finite time interval $\tau_{c} \approx \frac{\hbar E_{P}}{(\Delta E)^{2}} \approx 8 \times 10^{-4}$ s. Since the initial state $\left|K_{L}^{0}\right\rangle$ is a CP eigenstate, and the collapse states $\left|K^{0}\right\rangle$ and $\left|\bar{K}^{0}\right\rangle$ are not CP eigenstates, such collapse will be one main source of CP violation in the $K_{L}^{0}$ meson decay (Fivel, 1996a,b). However, considering the relativistic effect on the decay process of the high energy $K_{L}^{0}$ meson, such result may contradict the experimental observations, which show that there is no energy dependence of $\mathrm{CP}$ violation for $K_{L}^{0}$ decays in the energy range 1-200 GeV (Eidelman et al., 2004). In fact, since the states in each branch of the superposition are not spatially separated as in the common measurement situation, the difference of energy distribution may equal to the mass difference of $K^{0}$ and $\bar{K}^{0}$, which is $\Delta E<5 \times 10^{-10} \mathrm{eV}$ (Eidelman et al., 2004). It is this difference that determines the difference of space-times in the superposition. Then the collapse time should be $\tau_{c} \approx \frac{\hbar E_{P}}{(\Delta E)^{2}}>3 \times 10^{31} \mathrm{~s}$. This result is fully consistent with the experimental observations. 


\section{CONCLUSIONS}

Quantum measurement problem is the fundamental problem of quantum theory. Dynamical collapse theory is a promising way to solve this notorious problem. It considers the continuous Schrödinger evolution and the discontinuous quantum collapse as two ideal approximations of a unified evolution process. However, the physical origin of quantum collapse is still unknown. Feynman conjectured that the collapse of wave function may be induced by gravity. Along this line of reasoning, Penrose further strengthened the gravity-induced collapse argument. He argued that there is a profound and fundamental conflict between the general covariance principle of general relativity and the superposition principle of quantum mechanics, and the superposition of different space-times is physically improper. As a result, a quantum superposition of two space-time geometries, which corresponds to two macroscopically different energy distributions, should collapse after a very short time.

In this paper, we complete Penrose's argument by considering the discreteness of space-time. We show that Penrose's argument may fail in the continuous spacetime. The fundamental conflict between general relativity and quantum mechanics will require that the quantum superposition of different space-times can not exist at all, and should collapse instantaneously in the continuous space-time. This does not accord with experiment. However, such conflict can be reconciled in a consistent way in the discrete space-time. As a result, the wave function will collapse in a dynamical way. Moreover, we find that the minimum size of discrete space-time indeed yields a plausible collapse criterion consistent with experiments. By assuming that the source to collapse the wave function is the inherent random motion of particles described by the wave function, we further propose a concrete model of quantum collapse in the discrete space-time. It is shown that the model is consistent with the existing experiments and macroscopic experiences.

\section{REFERENCES}

Adler, S. L. (2001). preprint quant-ph/0109029.

Adler, S. L., Brody, D. C., Brun, T. A., and Hughston, L. P. (2001). Journal of Physics A: Mathematical and General 34, 8795.

Adler, R. J. and Santiago, D. I. (1999). Modern Physics Letters A 14, 1371

Amelino-Camelia, G. (2000). Nature 408, 661

Bell, J. S. (1993). In Davis, P. and Brown, J. (eds.), The Ghost in the Atom. Cambridge University Press, Cambridge.

Berg, B. A. (1996). preprint hep-ph/9609232.

Bunge, M. (1973). Philosophy of Physics. Reidel, Dordrecht.

Christian, J. (2001). In Callender, C. and Huggett, N. (eds.), Physics Meets Philosophy at the Planck scale. Cambridge University Press, Cambridge.

Cova, S., Ghioni, M., Lacaita, A., Samori, C., and Zappa, F. (1996). Applied Optics 35, 1956.

Diosi, L. (1989). Physical Review A 404, 1165. 
Eidelman, S., et al. (2004). (Particle Data Group). Physics Letters B 592, 1.

Einstein, A. (1936). Journal of the Franklin Institute 221, 378.

Feynman, R. (1995). In Hatfield, B. (ed.), Feynman Lectures on Gravitation, Reading, Massachusetts, Addison-Wesley.

Fivel, D. I. (1997a). Physical Review A 56, 146.

Fivel, D. I. (1997b). preprint quant-ph/9710042.

Garay, L. J. (1995). International Journal of Modern Physics A 10, 145.

Ghirardi, G. C., Pearle, P., and Rimini, A. (1990). Physical Review A 42, 78.

Ghirardi, G. C., Rimini, A., and Weber, T. (1986). Physical Review D 34, 470.

Heisenberg, W. (1957). Reviews of Modern Physics 29, 269.

Hughston, L. P. (1996). Proceedings of the Royal Soceity, London A 452, 953.

Pearle, P. (1989). Physical Review A 39, 2277.

Pearle, P. (1999). In Petruccione, F. and Breuer, H. P. (eds.), Open Systems and Measurement in Relativistic Quantum Theory. Springer Verlag, New York.

Pearle, P. (2004). Physical Review A 69, 42106.

Percival, I. C. (1994). Proceedings of the Royal Soceity, London A 447, 189.

Penrose, R. (1996). General Relativity and Gravitation 28, 581.

Polchinski, J. (1998). String Theory, Cambridge University Press, Cambridge.

Redhead, P. A. (1996). In Rigden, J. S. (ed.), Macmillan Encyclopedia of Physics, vol. IV, p.1657. Simon \& Schuster Macmillan, New York.

Rovelli, C. and Smolin, L. (1995). Nuclear Physics B 442, 593.

Salecker, H. and Wigner, E. P. (1958). Physical Review 109, 571.

Shan, G. (2000). Quantum Motion and Superluminal Communication, Chinese BT Publishing House, Beijing.

Shan, G. (2003). Quantum, Tsinghua University Press, Beijing.

Shimony, A. (1993). Search for a Naturalistic World View, vol. II. Cambridge University Press, Cambridge.

Smolin, L. (2001). Three Roads to Quantum Gravity, Weidenfeld and Nicolson and Basic Books, London and New York.

Snyder, H. S. (1947). Physical Review 71, 38.

von Neumann, J. (1955). Mathematical Foundations of Quantum Mechanics, Princeton University Press, Princeton. 